\title{
Application of the dynamic characterization of metals in automotive industry
}

\author{
Fabio D’Aiuto ${ }^{1, a}$, Daniele De Caro ${ }^{1}$, Claudio Federici ${ }^{1}$, Michele M. Tedesco ${ }^{1}$, Alessandro Ziggiotti ${ }^{1}$, and Ezio Cadoni ${ }^{2}$ \\ ${ }^{1}$ Centro Ricerche FIAT, 10135 Torino, Italy \\ ${ }^{2}$ DynaMat Laboratory, University of Applied Sciences of Southern Switzerland, 6952 Canobbio, Switzerland
}

\begin{abstract}
This paper presents the experimental methodology used by R\&D EMEA - Global Materials Labs Department to test metals at high strain rate of $500 \mathrm{~s}^{-1}$. The implementation of dynamic results in commercial FEM Software LS - DYNA for crash simulation are presented. The effects of the strain rate on the tensile properties of metals, used in automotive field, are evaluated using results obtained from a direct tension split Hopkinson bar, built in collaboration with the University of Applied Sciences of Southern Switzerland DynaMat Lab. Finally the complete mechanical characterization of the Magnesium alloy AZ31B is presented, from static up to dynamic tests, showing its applications in FCA (Fiat Chrysler Automobiles), problems and future developments.
\end{abstract}

\section{Introduction}

Mechanical behaviour of materials can change at high strain rates. Generally, by increasing the strain rate many materials show hardening effect, a decrease in failure strain and an increase in strength. This is a very important topic in the automotive field, where there are a lot of mechanical components subject to impulsive loads; automotive crashworthiness is one such example. Several vehicle components are designed to function as energy absorbers in the event of crash, such that the deceleration seen by the driver is not so harsh to cause severe bodily injury. In order to design structures able to absorb properly the energy, the material must be characterized in a way that reproduces the actual working conditions [1]. Therefore dynamic material testing method assuring results of high precision must be designed, in order to understand the strain rate sensitivity of materials.

It is scientifically well recognized that the most satisfactory testing method for accurate measurement of the dynamic mechanical properties of materials is the Hopkinson bar technique, that allows the generation of a loading pulse well controlled in rise time, amplitude and duration, giving rise to the propagation of an uniaxial elastic plane stress wave [2]. Different versions of the Hopkinson bar technique were developed to investigate dynamic tensile properties of materials. R\&D EMEA - Global Materials Labs Department, in collaboration with the University of Applied Sciences of Southern Switzerland DynaMat Lab, built a direct tension split Hopkinson bar, able to carry out high strain rate tension tests on metals in the range of $500-1500 \mathrm{~s}^{-1}$; it is the tension version of the Modified Hopkinson Bar developed by Albertini and Montagnani in seventies [3,4] and nowadays widely used [5-10].

\footnotetext{
${ }^{\text {a }}$ Corresponding author: Fabio.DAIUTO@crf .it
}

Experimental dynamic results are used in commercial FEM software to perform crash simulations. Indeed regarding the optimization of materials and structures in the body component, the finite element method has been considered a powerful tool to estimate crash performance [11]. Using FEM software the most important choice is the material model used to describe the flow behaviour of materials under various strain rates. There are several phenomenological and physics based constitutive models available in literature. Physic based model can provide more accurate representation of material behaviour over a wide range of temperature and strain rates, however they are not preferred because they require more data from some controlled experiments. For this reason phenomenological models are preferred in practical applications such as numerical simulations of components subjected to impulsive loads [12-14]. However, the researchers are continually studying and improving the material models in the numerical simulation due to the ever-increasing demands for more accurate predictions of dynamic material behaviour. The purpose of this work is to show the methodology used in FCA lead the dynamic characterization of metals and the implementation of these results in commercial FEM software for the crash simulation. Moreover, the complete characterization of the Magnesium alloy AZ31B will be presented, from static up to dynamic tests, showing its applications, problems and future developments.

\section{Mechanical testing at high strain rate}

The tensile tests at high strain rates are performed on the Modified Hopkinson Bar [3-10], installed at the GML laboratory. In Fig. 1 a sketch of the apparatus is shown.

The tensile load pulse is generated by releasing a certain amount of elastic mechanical energy stored in a portion of the input bar (pre-tensioned bar) through static 


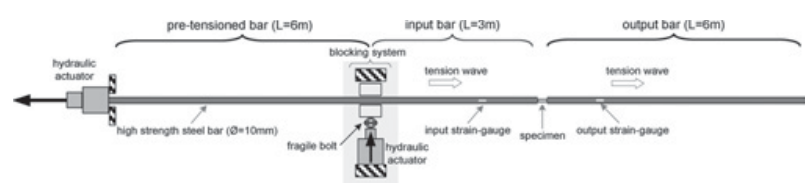

Figure 1. Scheme of the Modified Hopkinson Bar.

tensioning, up to a maximum stress value lower than its yield stress. At this scope a mechanical clamp [15] is used to grip the incident bar. The section of the incident bar between the clamp and the hydraulic actuator carries a static tensile load. The remainder of the input bar (to the right of the clamp) is unloaded. When the elastic mechanical energy is stored in the pre-tensioned bar and the specimen is inserted between the input and output bar, a notched bolt in the clamp is broken using a second hydraulic actuator, and an elastic tensile pulse is generated, propagating down the incident bar from the clamp toward the specimen. This pulse has a duration that is the double of the elastic wave travel time to move from the clamped section toward the free end of the input bar, and an amplitude that is half of the initial stored tensile pulse. As the wave reaches the input bar-specimen interface, a part of the pulse is reflected as a compression wave into the input bar and the remaining part is transmitted through the specimen. When the wave transmitted into the specimen arrives at the specimen-output bar interface, it is partially transmitted into the output bar and partially reflected into the specimen. If the specimen length is short so that the time taken by the wave to propagate through the specimen is short compared to the total time of the test, many reflections inside the specimen are created, allowing an homogenous stress and strain distribution along the specimen gauge length until fracture.

In this case the total length of the input bar is $9 \mathrm{~m}$, the portion of it that is pre-tensioned during the test is $6 \mathrm{~m}$, and the output bar length is $6 \mathrm{~m}$; both bars have a diameter of $10 \mathrm{~mm}$ and are made in high strength steel. This configuration permits to achieve tensile pulse duration of $2.4 \mathrm{~ms}$, allowing the deformation at constant high strain rate until fracture of high ductility specimens.

The semi-conductor strain-gage station is glued on the input bar at $750 \mathrm{~mm}$ from the specimen in order to record the deformation $\varepsilon_{\mathrm{I}}$ of the bar generated by the incident tension pulse during the propagation toward the specimen and the deformation $\varepsilon_{\mathrm{R}}$ caused by the part of the incident tension pulse reflected at the interface incident bar-specimen, reflection which is correlated with the deformation of the specimen. Another strain-gage station is glued on the output bar at the same distance from the specimen as the strain-gauge station on the incident bar; this second strain-gauge is used to record the deformation $\varepsilon_{\mathrm{T}}$ provoked on the bar by the part of the incident pulse which has been sustained by the specimen and has been therefore transmitted in the output bar; the transmitted pulse is proportional to the engineering stress in the specimen. A third strain-gauge station is bonded in the pre-tensioned bar to check the preload.

On the basis of the recorded signals $\varepsilon_{\mathrm{I}}, \varepsilon_{\mathrm{R}}$ and $\varepsilon_{\mathrm{T}}$, and applying the one-dimensional elastic plane stress wave propagation theory to the input bar-specimen-output bar

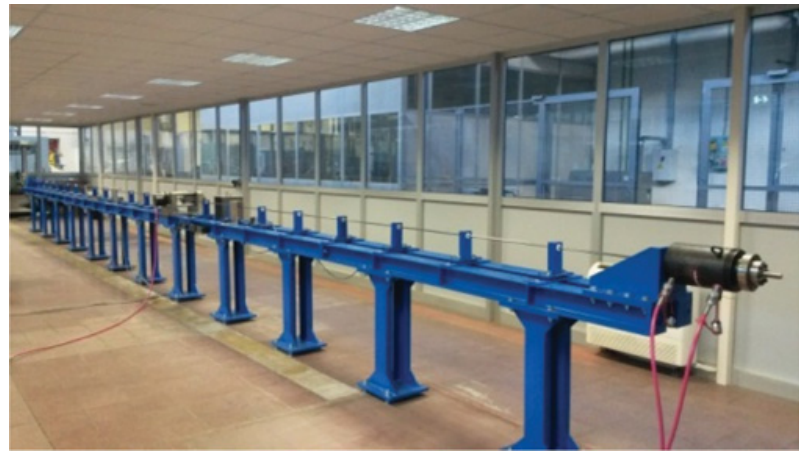

Figure 2. Modified Hopkinson Bar installed at the GML Laboratory.

system, it is possible to calculate the stress, strain and strain-rate in the specimen.

With the described apparatus it is possible to test a wide range of materials at high strain rate, in the range of 500 $1500 \mathrm{~s}^{-1}$. In Fig. 2 the Modified Hopkinson Bar installed at the GML Laboratory is shown.

\section{Crash simulation}

The main reason that pushes the automotive industries to investigate on the dynamic behaviour of materials is the need to perform crash test simulations. The objective of crashworthiness related design is to guarantee a prescribed safety level for the occupants during the impact events. In order to achieve it, it is necessary to design a vehicle structure collapsing in a controlled manner during the collisions to adsorb properly the impact energy created by the crash event. The underestimation or overestimation of strain rates in crashworthiness design may reduce the structure energy absorption capability, increasing occupant's body accelerations, resulting in more injuries.

\subsection{Numerical model}

In FCA the vehicle frontal crash simulations are conducted by LS-DYNA. To implement the mechanical behaviours of materials in commercial FEM software, the true stress plastic true strain data, from static and dynamic experimental results, are fitted by the following expression:

$$
\sigma_{T R U E}=\sigma_{Y}(\dot{\varepsilon})\left[1+C(\dot{\varepsilon}) \varepsilon_{T R U E} P L A S T I C\right]^{n(\dot{\varepsilon})} .
$$

Where $\sigma_{\mathrm{Y}}$ is the yield strength of materials. Only experimental results until the onset of necking are used. The constants $\mathrm{C}$ and $\mathrm{n}$ are calculated for each curve at different strain rate, respecting the Considère criterion in the necking point [16]:

$$
\frac{d \sigma}{d \varepsilon}=\sigma
$$

The equation is also extended in the field of large deformation. An example of the constitutive model at different strain rate is reported in Fig. 3.

This set of curves is built for each material used in FCA, and implemented in LS-DYNA to describe completely the mechanical behaviour of materials in a 


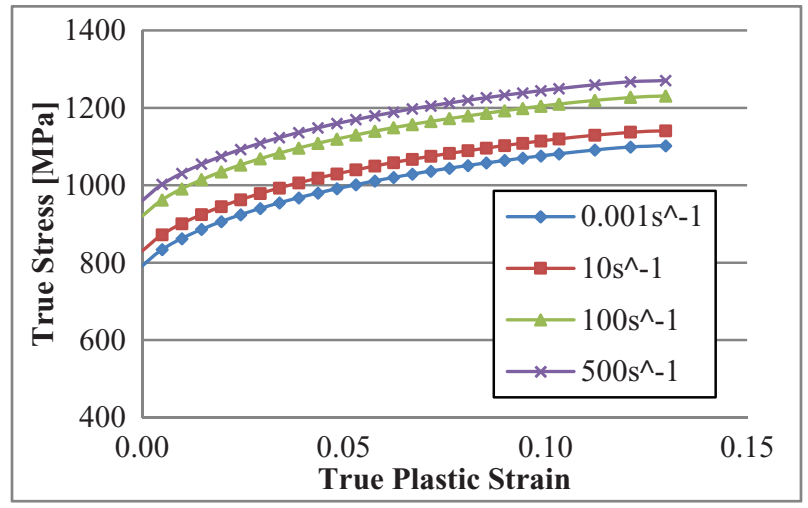

Figure 3. Constitutive model at different strain rate.

wide range of strain rate. This method is largely used in automotive impact engineering and is known as Piecewise Linear Plasticity. In LS-DYNA, a table is used to define for each strain rate value a load curve ID that gives the stress versus effective plastic strain. The lowest strain rate given in the table is applied if the strain rate falls below that minimum value. Likewise, the highest strain rate of the experimental data is used as a saturation plateau for the strain rate effects. In the simulation, the strain rate for each element is calculated and a linear interpolation between the experimentally determined strain rates is utilized to calculate the resulting stress in the plastic region. Moreover the simulations are conducted through dynamic explicit non-linear finite element model [17].

\subsection{Crash analysis}

Generally different types of crash analysis on BIW (body in white) are performed to test the safety of the vehicle:

- EURONCAP front crash, $40 \%$ overlap offset frontal crash at $64 \mathrm{~km} / \mathrm{h}$ into deformable barrier.

- USNCAP, $100 \%$ frontal crash at $56 \mathrm{~km} / \mathrm{h}$ into rigid barrier.

- Side impact test with moving deformable barrier, at $50 \mathrm{~km} / \mathrm{h}$, at 90 degree angle.

\section{Magnesium alloy characterization}

In an effort to improve the fuel efficiency of automobiles, car designers are investigating new material to reduce the overall vehicle weight. Magnesium alloys are good candidates to achieve that weight reduction due in part to their low density and high specific strength. Magnesium is the lightest structural metals with a density of approximately one-fourth that of steel and two-thirds that of aluminium, and as the specific strength and stiffness of magnesium exceeds that of most commonly used metals and some plastic-based metals, magnesium based materials are actively pursued by companies for weight-critical applications. Currently more than $60 \%$ of vehicle weight is due to use of steel or cast iron in the body structure, on the contrary, for example in America, the contribution to the overall vehicle weight of $\mathrm{Mg}$ alloys is only of $0.3 \%$ [18]. But bringing $\mathrm{Mg}$ parts to the market requires a several important study of its
Table 1. Chemical composition of AZ31B in wt.\%.

\begin{tabular}{|c|c|c|c|c|}
\hline Constituents & Al & Mn & Zn & Mg \\
\hline wt.\% & 2.8 & 0.31 & 0.5 & Balanced \\
\hline
\end{tabular}

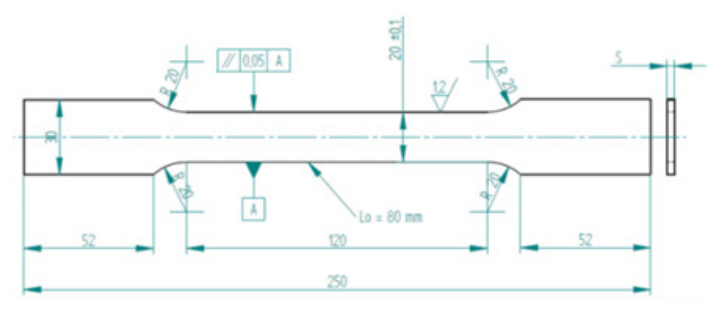

Figure 4. Static specimen geometry.

mechanical behaviour, its anisotropy, and it is also crucial to understand $\mathrm{Mg}$ alloys behaviour at high strain rates, especially in the automotive field where the components are often subjected to crash events.

Currently in literature there are few information about the tensile properties of $\mathrm{Mg}$ alloys at high strain rates, and the studies available are mostly on extruded and cast material, and predominantly in compression [19-22]. Ulacia et al. [19] performed an exhaustive testing campaign on AZ31-O sheet at dynamic $\left(\dot{\varepsilon} \sim 10^{3} \mathrm{~S}^{-1}\right)$ and low $\left(\dot{\varepsilon} \sim 10^{3} \mathrm{~S}^{-1}\right)$ strain rates, in tension and compression. He showed the different microstructural evolution at high strain rates and high temperatures. J. Xiao et al. [20] carried out impact test on AZ31 Mg alloy reinforced by $1 \%$ vol silicon carbide nanoparticles, showing strong rate dependence, increasing in flow stress as the strain rate increases. Hasenpouth [22] performed tensile test on AZ31B magnesium alloy sheets, at low, medium and high strain rates. He found a clear increase in tensile strength as the strain rate increases, showing a stress rise of approximately $60 \mathrm{MPa}$.

The complete characterization of the Magnesium alloy AZ31B is presented, from static up to dynamic tests, realized by the GML Laboratory in collaboration with SUPSI.

\subsection{Material}

Nowadays AZ31B is the most common commercial magnesium alloy available in sheet form. It was obtained from Elektron, whose chemical composition in wt\% is given in Table 1.

\subsection{Quasi Static Test}

The quasi static test $\left(\dot{\varepsilon} \sim 10^{3} \mathrm{~S}^{-1}\right)$ were performed by means of an electromechanical universal testing machine, with $200 \mathrm{kN}$ maximum load bearing capacity, installed at GML Laboratory. In order to measure the strain on the specimen gauge length a video extensometer was used. The sample geometry is reported in Fig. 4. The tests were carried out on specimens oriented in longitudinal, transversal and diagonal directions respect to the rolling direction.

In Figs. 5 and 6 the engineering and true curves along the three orientations considered are reported. 


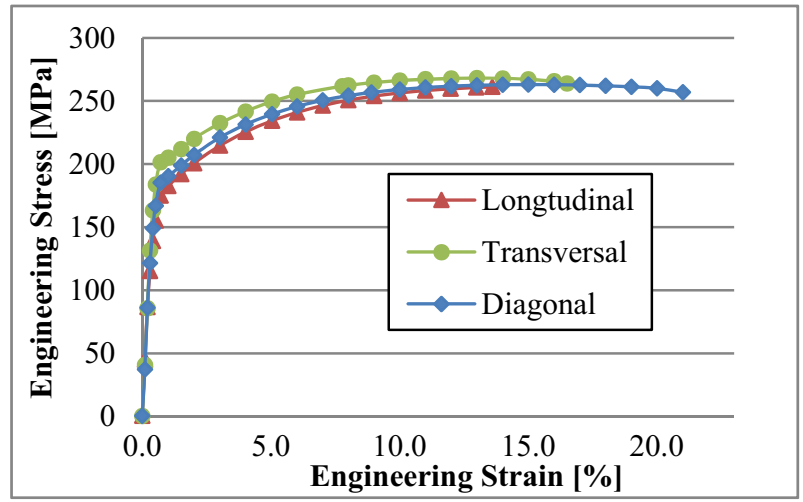

Figure 5. Static engineering stress vs. strain curves for three orientations.

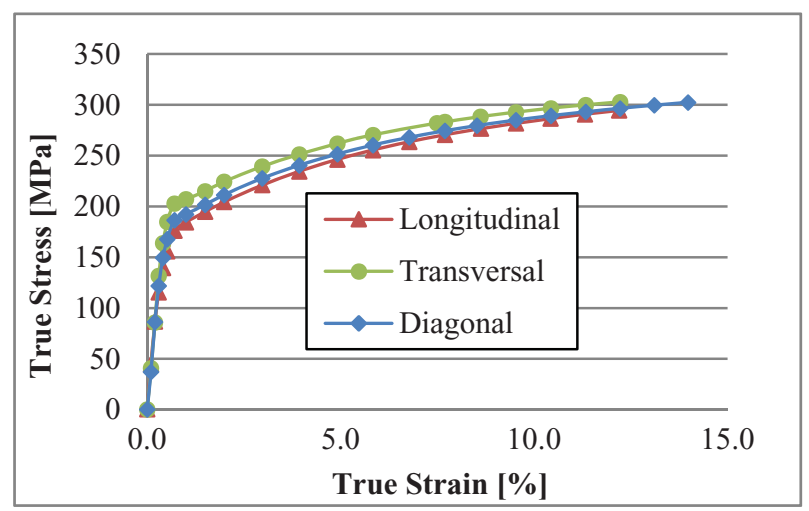

Figure 6. Static true stress vs. strain curves for three orientations.

As shown in the figures above, the mechanical properties of material along the three directions are slightly different, both in elongation and flow plastic stress, attesting the anisotropy of magnesium alloys. However the dynamic tests were carried out only on specimens oriented along the rolling direction.

\subsection{Dynamic test}

Dynamic tensile tests on AZ31B alloy were conducted at medium $\left(5-50 \mathrm{~s}^{-1}\right)$ and high $\left(500 \mathrm{~s}^{-1}\right)$ strain rate.

The medium strain rate tests were performed by SUPSI on Hydro-pneumatic machine (HPM) [6,7,7,10].

The high strain rate tests, at $500 \mathrm{~s}^{-1}$, were carried out on the Modified Hopkinson Bar installed at GML Laboratory.

The specimens tested were flat, having $10 \mathrm{~mm}$ gauge length, $4 \mathrm{~mm}$ width and $1.5 \mathrm{~mm}$ thickness; the geometry is the same reported in $[6,10]$. The experimental data were analysed to obtain the stress versus strain curves at three different strain rate regimes. The engineering stress versus engineering strain curves and true stress versus true strain curves of $\mathrm{Mg}$ alloy are compared in Fig. 7 and Fig. 8, respectively. The true curves were plotted until the neck point for each strain rate regime. Related to the quasistatic results, for comparison only the true stress versus true strain curve, due to the different geometry used in quasi-static and dynamic tests, is reported.

The results clearly show that the AZ31B alloy is very sensitive to the strain rate; in fact the flow stress increases

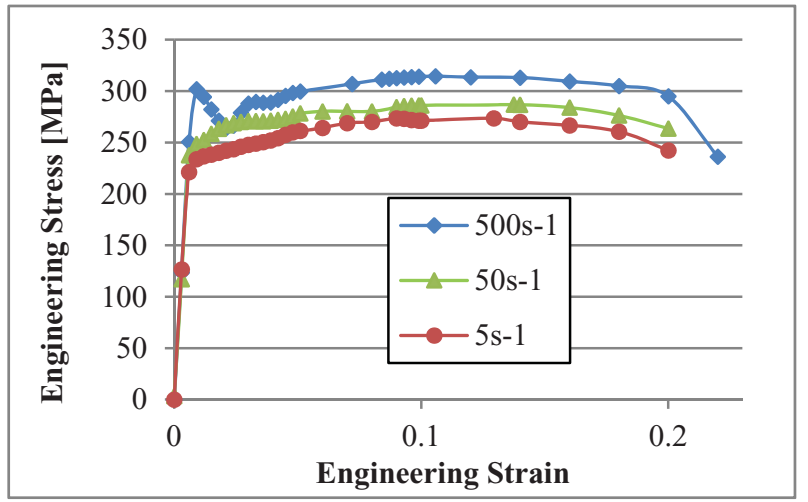

Figure 7. Dynamic engineering stress vs. strain curves for three strain rates.

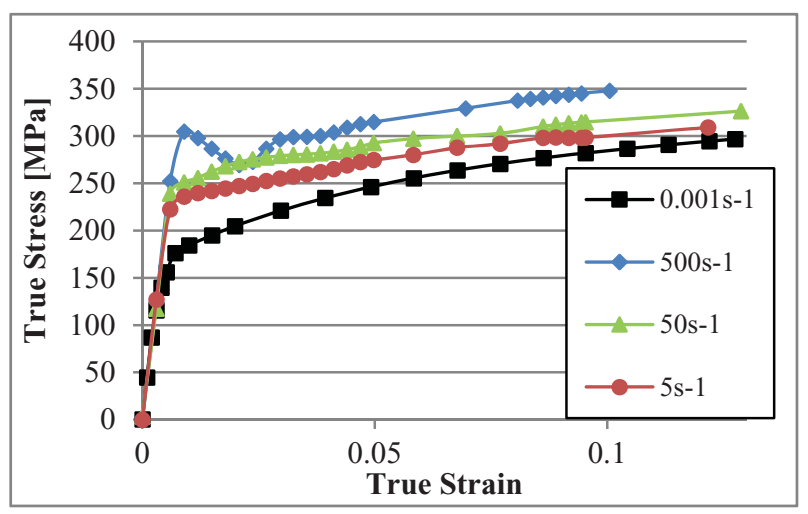

Figure 8. Dynamic true stress vs. strain curves for three strain rates.

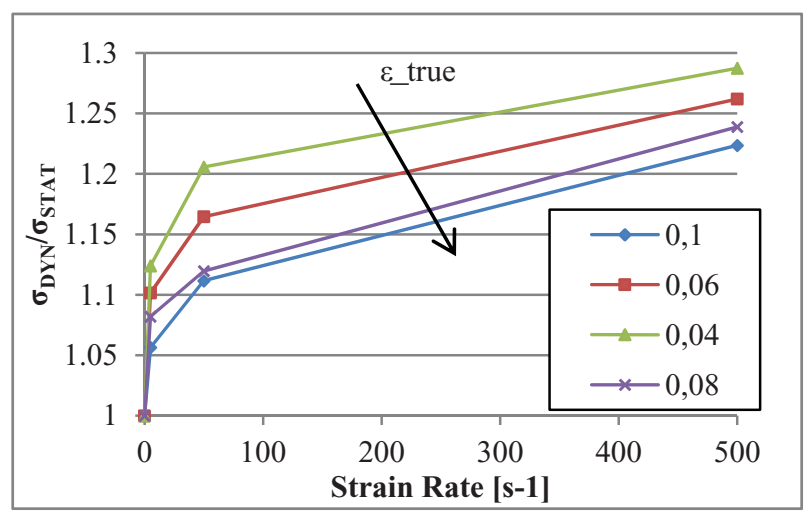

Figure 9. DIF vs. strain rate curves, at four different true strains.

as the strain rate rises from 0.001 up to $500 \mathrm{~s}^{-1}$. Moreover the fracture engineering strain is almost the same as strain rate increases, so the area under stress-strain curve is more at high strain rate, increasing the fracture energy and toughness of material.

To better understand the effect of the strain rate on mechanical behaviour of $\mathrm{Mg}$ alloy, in Fig. 9 the dynamic stress-static stress ratio versus strain rate for four different true strains is plotted. The figure shows an increasing in strain rate effect as the strain rate increases, but it is also possible notice that the strain rate sensitivity on $\mathrm{Mg}$ alloy seems decrease as the true strain increases. This particular behaviour of $\mathrm{Mg}$ alloy is better represented in Fig. 10 


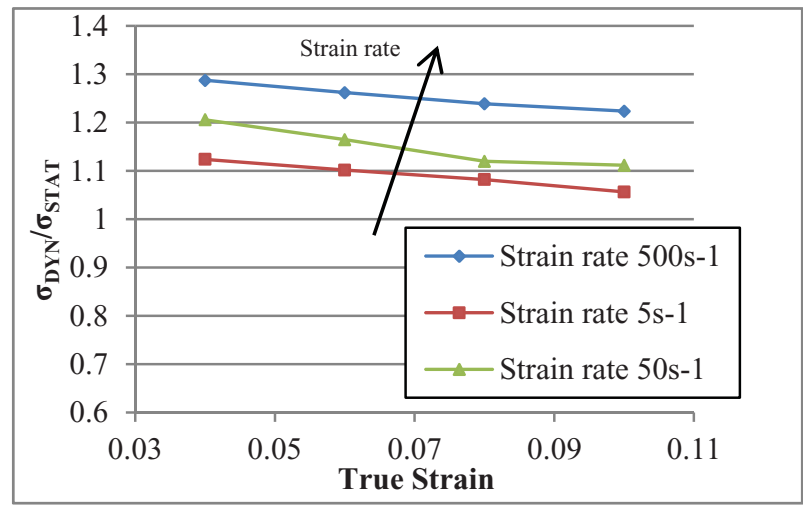

Figure 10. DIF vs. true strain curves, at three different strain rates.
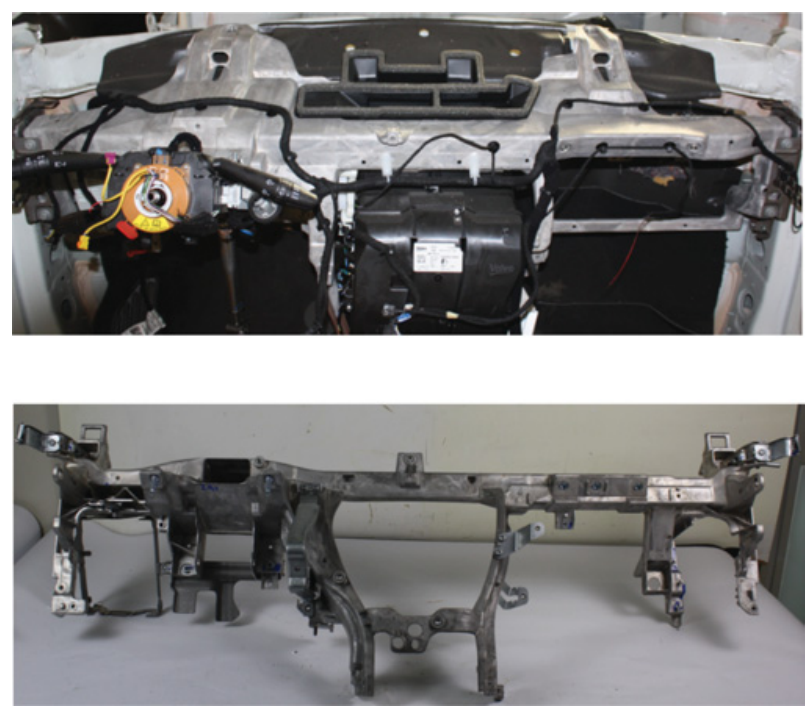

Figure 11. Instrument Panel Beam in $\mathrm{Mg}$ alloy.

that plots the dynamic stress-static stress ratio (known as Dynamic Increase Factor - DIF) versus true strain for three different strain rates.

\subsection{Applications}

FCA in the last years has increased the usage of Magnesium alloys in its products in order to perform a significant weight reduction of the vehicle. The main applications of $\mathrm{Mg}$ alloys are currently related to the instrument panel beam, the seat structure, gearbox and steering wheel, as depicted in Fig. 11.

At the moment the $\mathrm{Mg}$ alloys are used only as high pressure die casting (HPDC) and not in sheet form, for their actual manufacturing difficulties. Unfortunately, due to their crystallographic structure and texture, magnesium alloys exhibit low ductility at room temperature and strong anisotropy in their constitutive behaviour. Therefore, elevated temperature stamping is needed to produce magnesium alloy parts, which increases their production cost. Improvements in magnesium alloy sheet are thus needed and research interest on this activity has greatly increased in the past few years.

\section{Conclusions}

The experimental methodology used by GML to test materials at high strain rates is presented in this paper. Moreover the dynamic results implementation for crash simulation is described. The complete mechanical characterization of AZ31B Magnesium alloy is investigated. The quasistatic tests are performed along three different orientations, attesting the anisotropy of magnesium alloys. The results obtained from dynamic tests show the strain rate effect on material, that exhibits an increasing in strength as the strain rate rises from 0.001 up to $500 \mathrm{~s}^{-1}$; on the contrary the engineering fracture strain is almost the same at different strain rates, resulting in enhancement of energy adsorbed at fracture.

In conclusion, due to the increasing demand for cutting down energy consumption and greenhouse gas emissions, the $\mathrm{Mg}$ alloys are a good choice in order to reduce the overall weight of the vehicle for more economic use of fuel. However nowadays the good mechanical behaviour of $\mathrm{Mg}$ alloys in sheet form, and its advantages for weight-critical applications, are obstructed by their actual manufacturing difficulties.

\section{References}

[1] A.T. Owens, Strain response of particulate composites under high rates of loading, MS Thesis, Auburn University, Alabama (2007).

[2] H. Kolsky, Proc. Phys. Soc. Sect.B62, 676-700 (1949).

[3] C. Albertini, M. Montagnani, Nucl. Eng. Des. 37, 115-124 (1976).

[4] C. Albertini, M. Montagnani, Inst. of physics conf. series 21, 22-32 (1974).

[5] E. Cadoni, M. Dotta, D. Forni, P. Spätig, J. Nucl. Mat. 414(3), 360-366 (2011).

[6] N.K. Singh, E. Cadoni, M.K. Singha, N.K. Gupta, Mat. Des. 32, 5091-5098 (2011).

[7] E. Cadoni, L. Fenu, D. Forni, Constr. Build. Mat. 35, 399-407 (2012).

[8] E. Cadoni, M. Dotta, D. Forni, N. Tesio, C. Albertini, Mat. Des. 49, 657-666 (2013).

[9] C. Albertini, E. Cadoni, G. Solomos, Phil. Trans. R. Soc. A 2014 372, 20130197.

[10] E. Cadoni, F. D’Aiuto, C. Albertini, Proc. DYMAT2009, EDP Sciences, 1, 135-141 (2009).

[11] A.K. Pickett, T. Pyttel, F. Payen, F. Lauro, N. Petrinic, H. Werner, Int. J. Impact Eng. 30, 853-872 (2004).

[12] F.J. Zerilli, R.W. Armstrong, J. Appl. Phys. 61, (1987).

[13] G.R. Cowper, P.S. Symonds, Brown University Applied Mathematics Report 28, 1-46 (1958).

[14] G.R. Johnson, W.H. Cook, Eng. Fract. Mech., 21, 31 (1985).

[15] M. Montagnani, C. Albertini, U. Buzzi, M. Forlani, 1973 Dispositif de contrainte a accumulation mecanique pour essais dinamique de traction. Institut national de la propriete industrielle: Paris Brevet No. 74.17085. The Patent Office of London, Patent No. 1.473.683, 1974. Italian Patent No. 50008A. 
[16] K.S. Havner, Int. J. Plast. 20, 965-978 (2004).

[17] Z. Shen, X. Qiao, H. Chen, Proceedings of the FISITA 2012 World Automotive Congress, Vol. 9, Automotive Safety Technology.

[18] G.S. Cole, A. North, American Automotive Strategic Vision of Magnesium, USAMP report 2007.

[19] I. Ulacia, N.V. Dudamell, F. Galvez, S. Yi, M.T. Perez-Prado, I. Hurtado, Act. Mat. 58, 2988-2998 (2010).
[20] J. Xiao. D.W. Shu, X.J. Wang, Int. J. Mech. Scie. 89, 381-390 (2014).

[21] M.T. Tucker, M.F. Horstemyer, P.M. Gullett, H. El Kadiri, W.R. Whittington, Scr. Mater 60, 182-185 (2009).

[22] D. Hasenpouth, Tensile high strain rate behaviour of AZ31B Magnesium Alloy Sheet, (2010).

[23] E.D.H. Davies, S.C. Hunter, J. Mech. Phys. Solids 11, 155-179 (1963). 